\title{
Acute coronary syndrome in cancer patients. Part II: invasive and conservative treatment options, takotsubo syndrome problem
}

\section{Grzegorz Piotrowski}

Department of Cardio-Oncology,

Medical University of Lodz, Poland

Department of Cardiology,

Provincial Multidisciplinary Oncology and Traumatology Center Nicolaus Copernicus, Lodz, Poland

Correspondence:

Grzegorz Piotrowski Department of Cardiology, Provincial Multidisciplinary Oncology and Traumatology Center Nicolaus Copernicus 91-513 Lodz, Poland, Pabianicka 62 e-mail:gpiotr4@wp.pl phone: +48607244594 fax: +48426895491

Received: 5.09.2020 Accepted: 30.10 .2020

\section{ABSTRACT}

Acute coronary syndrome (ACS) and oncological disease are more frequently observed in the general population as discussed in the part I of this article. Treatment of myocardial infarction in oncological patients becomes a real struggle for clinicians, especially that the data from clinical trials including cancer patients with ACS are very limited. The choice of treatment modality should consider many existing factors considering the type of ACS - non-ST-segment elevation myocardial infarction vs ST-segment elevation myocardial infarction, patient's condition, type of cancer and oncological treatment applied. Taking into consideration above mentioned factors, clinicians have to face three therapeutic options: invasive, conservative or combination of both in order to choose the best and most beneficial treatment. This article summarizes the current therapeutic approach to the management of ACS in cancer patients.

Key words: cardio-oncology, acute coronary syndrome, treatment strategies 


\section{ACUTE CORONARY SYNDROME TREATMENT OPTIONS - INVASIVE OR CONSERVATIVE?}

Treatment strategy of a patient with both acute coronary syndrome (ACS) and neoplastic disease is not based on the knowledge gained from the clinical trials. There is practically no data from randomized clinical trials in this group of patients. Importantly, current guidelines and recommendations are based on the results of the clinical trials that do not apply to the population of cancer patients. It should be recalled that neoplasmatic condition was the primary exclusion criteria from the most important studies, therefore, this group of patients were not recruited. Therefore, in the documents systematising the knowledge on the management of cardiovascular diseases in cancer patients, the recommendations have level C, i.e. they are a consensus of experts based on their experience. The available results from clinical trials and the guidelines based on them cannot be automatically applied to this population. The decision on diagnostic and therapeutic procedures in this population must be individual in each case with taking into consideration general standards.

The mainstay of ACS treatment is coronary revascularization. In the recent past, fibrinolytic therapy was used to dissolve the thrombus in ST elevation myocardial infarction (STEMI) and with the image of a new left bundle branch block (LBBB). Today it is known that the most effective method of treating ACS, especially myocardial infarction, is coronary angioplasty, which usually ends with the implantation of a stent into the coronary artery [1-3].

Selecting an ACS treatment method in an oncological patient is difficult and should always be individual. Due to the many comorbidities the assessment of the benefit-risk ratio is complex and often not obvious. Choosing adequate treatment modality in ACS in this situation should always include following factors:

1. Cancer-related factors

2. Patient's criteria

3. Currently used oncological treatment strategy

4. Prognosis

5. STEMI vs. NSTEMI.

\section{Cancer-related factors}

When considering cancer-related factors, the following should be taken into account: the histological classification of cancer, cancer staging (i.e. limited disease, with lymph node involvement, advanced - with distant metastases). Each tumor has a distinct biology that affects the clinical course of ACS. Patients with myocardial infarction and lung cancer have a significantly higher mortality, whereas those with active colorectal cancer are at higher risk of bleeding; the best prognosis is observed in breast cancer, especially in the limited disease. Some hematological neoplasms are characterized by a very high bleeding tendency. As mentioned before the stage of cancer is of great importance. In general, neoplasms with distant metastases have worse prognosis, but there is also a large variation depending on the histological type of cancer. Patients with metastatic cancer of the colon, lung and prostate have a very high in-hospital mortality in ACS [4]. It also matters whether the cancer is in an active phase or in remission and whether the patient underwent successful oncological treatment. The ACS prognosis is worse in newly diagnosed neoplasms inter alia for the reason that in the first weeks after cancer diagnosis, a very strong prothrombotic state is observed. It should be noted that even patients who are cured of neoplastic disease have worse early and late ACS prognosis when compared to those with no cancer history [4].

Patients with a good clinical prognosis (breast, prostate and colorectal cancer) should rather be treated invasively. Similarly, in the case of a limited neoplastic disease - without distant metastases, the early invasive strategy should be considered. It needs to be highlighted that even patients with advanced neoplastic disease who undergo coronary angioplasty have lower in-hospital mortality and lower mortality within one year [5]. In the retrospective observation of Yusuf et al., the lack of adequate treatment of myocardial infarction in patients with cancer, regardless of its stage, led to a mortality of $74 \%$ within one year [6].

2. Criteria related to the patient

A large proportion of cancer patients meet the criteria of "frailty syndrome". Their bodies are very susceptible to disturbances of unstable homeostasis due to relatively mild disease factors. The cancer patient is usually older, often diagnosed with more than one disease. Usually these are kidney failure, liver damage, heart failure, diabetes, atrial fibrillation. Disorders such as anemia, thrombocytopenia, dyselectrolytemia, acid-base imbalance, coagulation disorders are often present. Characteristically, the patient may present symptoms such as diarrhea, nausea, vomiting and fever. Particular attention should be paid to the kidney function (GFR, glomerular filtration) in the context of the use of nephrotic contrast and the use and dosing of many drugs.

\section{Oncological treatment strategy}

The factor that plays a significant role in the choice of ACS therapy is the currently used or planned oncological treat- 
ment. If a patient suffers a myocardial infarction while on anthracycline therapy, thrombocytopenia is to be expected in the coming days. If a diagnostic procedure (biopsy) or urgent surgery to remove a confined tumor is planned, then implantation of a coronary stent that requires dual antiplatelet therapy (DAPT) will postpone invasive diagnosis or oncological treatment. Consequently this may result in the spread of the cancer disease.

\section{Prognosis}

The prognosis is a very important factor in choosing treatment strategy. Patients in the final stage of an incurable cancer, whose survival is short, are not considered as good candidates for aggressive invasive treatment of ACS. Such patients will not benefit and will be exposed to complications of the procedure: bleeding, stent thrombosis, kidney damage, infection. On the other hand, in the case of a patient with a potentially curable neoplastic disease or in permanent remission, without severe metabolic disorders, there is no reason to give up on the best method of ACS treatment - coronary angiography and coronary angioplasty.

\section{STEMI vs. NSTEM}

In the situation of ACS manifested by STEMI invasive treatment is always preferred. In turn, in ACS NSTEMI there is more time to assess the benefits and risks of a particular patient. This applies to situations where we do not observe an unstable clinical state. In these cases, one should rather follow the principles recommended by the guidelines [2], also taking into account the above-mentioned conditions. The high probability of a type 2 infarction should firstly direct therapeutic interventions that remove the factors responsible for the oxygen deficit in the myocardium.

\section{INVASIVE TREATMENT}

The choice of invasive treatment should consider the cancer-related factors that will modify implemented management. Vascular access from the radial artery should be the strategy of first choice. It is associated with fewer complications (i.e. bleeding, especially at the access site, hematomas, infections). It provides the patient with better comfort, does not embarrass the patient and enables faster convalescence. Studies have shown that this type of vascular access is associated with significantly lower mortality. Radial access should be a priority especially in thrombocytopenia and in the presence of bleeding disorders. Contraindication to this access is collateral insufficiency of the punctured upper limb and a dialysis fistula [7]. Complications of femoral puncture include retroperitoneal bleeding, pseudoaneurysm of the femoral artery, fistula, prolonged bleeding that may lead to shock, delayed epithelialization after the use of closure tools. All of these events are more likely in a patient with cancer. Bleeding into the retroperitoneal space is particularly dangerous, especially at a high puncture point, which is extremely difficult to stop in a patient with thrombocytopenia, during dual antiplatelet therapy and after loading dose of heparin [8].

A special situation is the need for coronary angiography in a woman with breast cancer after mastectomy on the vascular access side. There are no rational reasons to deprive the patient of a much better and safer performance of radial angiography/ angioplasty in such situations. In a retrospective analysis of 129 patients from two centers in the USA and Canada, who underwent 42 procedures from the radial approach on the same side and 92 from the contralateral approach or from the femoral artery, it was not found that the radial approach on the side of the mastectomy performed was connected with swelling of the upper limb. Also, for pathophysiological reasons, complication of puncture of the radial artery in the form of closure of this artery, which happens in $20 \%$ of cases, cannot cause upper limb edema. Swelling of the upper limb results from obstruction of the outflow of lymph as a result of fibrosis of the axillary lymph vessels after removal of lymph nodes from this region [9]. However, if possible, it is best to select the radial artery on the opposite side of the mastectomy or to use the ulnar access $[10,11]$.

The next decision the interventional cardiologist has to make is when choosing the optimal coronary stent. Most of the documents relating to the issues of coronary interventions in cardio-oncological patients recommend the implantation of bare metal stent (BMT) $[8,12,13]$. The 2018 guidelines of the European Society of Cardiology and Cardio-Thoracic Surgery (ESC; EACTS) recommend the use of drug eluting stents (DES) in all patient populations [7]. The author shares this opinion also in relation to patients with ACS and accompanying cancer. However, in the case of cardio-oncological patients, a third-generation stent should be used, which requires the shortest possible administration of dual antiplatelet therapy DAPT and is associated with a lower risk of thrombosis. The author, based on the product characteristics, suggests the use of the following stents: SYNERGY ${ }^{\circledR}$ (everolimus), Resolute Onyx ${ }^{\circledR}$ (zotarolimus), Biofreedom $^{\circledast}$ (biolimus), Ultimaster ${ }^{\circledast}$ (sirolimus). An oncological patient will always encounter the problem of thrombocytopenia in the course of the disease. Due to the fact that this group of patients will often require urgent chemotherapy leading to a reduction of platelet concentration, or urgent diagnostics, even surgery, 
in selected cases, when technical conditions allow it, the treatment could be limited to coronary balloon angioplasty without stent implantation. In these cases the use of a drug-coated balloon (DCB) technique should then be considered, especially for interventions in peripheral arteries. Such a strategy will allow for administration of DAPT for only 2 weeks and the continuation of diagnostics and resumption of oncological treatment. Urgent oncological procedures and chemotherapy are recommended to be postponed for at least 4 weeks after ACS [14]. The strategy of using DCB alone seems reasonable if angioplasty needs to be performed urgently while oncological drugs administration, the use of which should not be discontinued and its continuation is associated with the risk of thrombocytopenia [15]. After the oncological procedures are completed and the cancer disease is stable or cured, it is possible to resume elective surgery on the coronary vessels and then implant a stent in the place of the earlier angioplasty. Such a long-term strategy is called "multi-step" or "hybrid" [8].

Due to high thrombogenicity and high risk of stent thrombosis, unnecessary "stenting" of coronary bifurcation lesions and "overlap" stent implantation should be avoided $[8,13]$. It also seems reasonable to use aspiration thrombectomy more often than in the general population, despite the relatively low class of recommendations for this technique. The author has often encountered large-size blood clots in the vessels without severe atherosclerotic lesions. Not all coronary lesions should be "stented", but apart from the lesion responsible for myocardial infarction, really significant, symptomatic stenosis should be selected for the next urgent stages, especially if leaving them could limit life-saving oncological treatment. The fractional flow reserve (FFR) method should play a huge role in the qualification for the next stages of angioplasty in this group of patients. If a decision is made to treat subsequent lesions, the use of intravascular ultrasound (IVUS) and optical coherence tomography (OCT) should be considered to optimize the stent application [12]. Intravascular imaging methods allow for better preparation for the procedure, familiarization with the anatomy of the intervention site, selection of the appropriate stent, avoiding dissection, optimizing stent implantation and avoiding complications of the procedure, including reducing the risk of restenosis [12].

Sometimes the need for urgent oncological intervention (chemotherapy, diagnostics, surgical treatment) should, in justified and often controversial situations, give priority to surgical revascularization, after which there is no absolute need for DAPT to prevent in-stent thrombosis. Usually 2 to 4 weeks after revascularization surgery, oncological diagnostic and therapeutic procedures can be resumed. Of course, this time will depend on the time of recovery after surgery.

Coronary artery bypass graft surgery (CABG) can be safely performed at platelet levels $>50,000,000 / \mathrm{ml}$. CABG will be considered rather not when treating the lesion directly responsible for acute ischemia, but when there is an absolute need for revascularization of subsequent critical changes in the coronary arteries. Surgical revascularization may be considered in patients in good condition, without the burdens that increase the risk of surgery, and with a good oncological prognosis and a potentially curable tumor. CABG is especially indicated if surgery or oncological diagnostics cannot be postponed due to the very high tumor expansion and the risk of rapid tumor spread (e.g. lung cancer) [16]. If the patient has indications for urgent oncological surgery, surgical revascularization may be performed simultaneously or in two stages, which allows to avoid delaying urgent treatment of a cancer [12]. As a rule, however, the oncological patient is a patient with the frailty syndrome and will not be a good candidate for CABG. Most cancer patients will be revascularized by coronary angioplasty [8].

Invasive coronary and surgical procedures on the coronary arteries in patients after radiotherapy may be difficult due to the severe fibrosis and sclerosis of the atherosclerotic lesions following radiation. Also, the thoracic tissues and internal arteries may be fibrotic following irradiation, which makes surgical revascularization difficult and may complicate wound healing $[8,13,16]$.

One of the greatest challenges in catheterization and revascularization in cancer patients is thrombocytopenia, which is very common in this group of patients. Most patients with blood cancers and solid tumors have thrombocytopenia at some point in their disease. It may be part of the clinical picture of neoplastic disease or a complication of chemotherapy [17]. Preventive transfusion of platelets up to $10,000 / \mathrm{ml}$ is not generally recommended in non-bleeding patients. Exceptions are necrotic tumors, bladder cancer during treatment, female reproductive system tumors, colorectal cancer and melanoma, and patients with fever. In these cases, even in the absence of symptoms, platelet transfusions are recommended at levels lower than $<20,000 / \mathrm{ml}$ [18]. Diagnostic coronary angiography appears to be safe at a platelet level of $10,000 / \mathrm{ml}$. Then one of the antiplatelet drugs - acetylsalicylic acid, can be safely used. Coronary angioplasty can be performed if the platelet level is $30,000 / \mathrm{ml}$ or more $[12,18]$. DAPT may be used at this level, too. Between 10,000 and 30,000 , due to the inability to use DAPT safely, only coronary angioplasty should be considered, if possible. A DCB technique may be considered. If the 
platelet level is $<50,000 / \mathrm{ml}$, it is recommended to use a reduced dose of bolus of unfractionated heparin during coronary angiography - 30-50 U/kg, at the same monitoring the activated clotting time (ACT) and maintaining its value $>250 \mathrm{~s}$. In angioplasty, it is recommended to use DAPT for 2 weeks, 4-6 weeks after BMS implantation and about 6 months after DES implantation [12-14]. Higher generation stents are already available for which the DAPT time can be shortened. The stent, as a foreign body in the coronary artery, activates platelets, which leads to the formation of a clot in the stent. The stent is gradually endothelialized that is, the endothelial cells adhere to the metal surface of the stent. This causes the endothelial cells to fully cover the metal surface of the stent and restore proper communication between the platelets and the vessel surface, which prevents thrombosis. Until endothelialization is complete, the action of platelets should be inhibited, which is achieved by DAPT. Therefore, such procedures should be implemented in cancer patients. Despite the use of DAPT, cancer patients have a greater risk of stent thrombosis than patients without cancer [19]. Since anticancer drugs inhibit cell proliferation, that is, cell division, there are indications that when used soon after stent implantation, some antineoplastic drugs may inhibit endothelialization, potentially prolonging the duration of DAPT use [20].

DAPT therapy should often be discontinued in cardiovascular patients before the minimum time needed for endothelialization has elapsed. The results of IVUS or OCT can be helpful in order to make the difficult decision about early DAPT termination. The second method allows for the visualization and assessment of the degree of stent endothelialization stenting, evaluation of the correctness of the coronary stent position and the presence of restenosis. It has been shown that OCT enables the precise identification of patients in whom DAPT can be safely terminated before the recommended time [21].

\section{PHARMACOLOGICAL TREATMENT}

The gold standard of ACS treatment is mechanical revascularization - coronary angioplasty with stent implantation [3], but its success depends on properly conducted and optimal pharmacological therapy. The scheme of such therapy is very well known in the general population. However, in cancer patients, pharmacological treatment is a real challenge. Clinicians meet many disorders that limit the use of most drugs. There is also no experience from controlled, randomized clinical trials for this group of patients.
There appears to be no room for thrombolytic therapy in patients with active cancer who develop ST-segment elevation myocardial infarction during treatment. Although there are cases in the literature, in which this treatment was used in myocardial infarction in cancer patients [6]. The high frequency of such treatment was due to the lack of access to hemodynamic centers [6]. The use of thrombolytic treatment in neoplastic disease is complicated by disorders in the coagulation system and frequent thrombocytopenia [22, 23]. Of course, an absolute contraindication to thrombolytic therapy is active bleeding and neoplasms of the central nervous system.

DAPT is a very difficult issue from the point of view of clinical practice. It has been shown that the use of acetylsalicylic acid in patients with cancer and thrombocytopenia $(<50,000)$ was associated with significantly lower mortality after 7, 30 days and one year compared to no antiplatelet therapy [24]. In cancer patients, clopidogrel is rather recommended as the second component of DAPT, with a lower risk of bleeding complications compared to the higher generation $\mathrm{P} 2 \mathrm{Y} 12$ inhibitors (ticagrelor, prasugrel) for whom no safety data are available at platelet levels below $<50,000 / \mathrm{ml}$. The author suggests that in a patient with a low bleeding risk, stable course of neoplastic disease, a higher generation P2Y12 inhibitor may be considered, which is characterized by a potentially stronger antiplatelet effect translating into greater clinical benefits $[25,26]$, which is important in the context of high thrombogenicity in cancer patients. There are single reports in the literature where prasugrel increased the risk of metastasis in colorectal cancer [27].

Due to the high risk of bleeding in this group of patients, the use of intravenous antiplatelet drugs: cangrelor or glycoprotein (GP) Ilb/Illa inhibitors (eptifibatide, tirofiban) may be considered in the first days of myocardial infarction treatment. The short halflife of these drugs makes it possible to stop their action immediately and temporarily in the event of bleeding. These drugs can also be used in the perioperative period if urgent surgery is needed soon after stent implantation [7]. The fact is that cancer patients have an increased risk of both bleeding and clotting. If the risk of stent thrombosis is particularly high and at the same time the risk of bleeding is low, then the ATLAS II study strategy is worth considering. The strategy suggests use of a low dose of rivaroxaban $2 \times 2.5 \mathrm{mg}$ added to DAPT containing clopidogrel after stent angioplasty procedures. Such treatment reduced the risk of stent thrombosis and reduced the composite endpoint of cardiovascular deaths, myocardial infarctions, and strokes [28]. Such a recommendation may seem justified in selected patients in the context of the American recommendations regarding the 
primary prevention of venous thromboembolism in outpatients undergoing chemotherapy [29]. However, it should be remembered that the new oral anticoagulants/direct oral anticoagulants (NOAC/DOAC) doses recommended for the prevention of venous thromboembolism are higher - for rivaroxaban $10 \mathrm{mg}$ once a day.

It must not be forgotten that DAPT has two goals. One is the prevention of stent thrombosis, the other is the prevention of recurrent vascular events, not only coronary, but cerebral and other peripheral arteries [30]. The first goal is usually achieved after 3 months with the third generation stents available today, and the second goal, on the other hand, seems to be extremely relevant, especially in oncological patients. Stable neoplastic disease in remission, as a chronic inflammatory disease, probably intensifies remodeling and accumulation of atherosclerotic plaques. Therefore, if a patient with cancer is stable, has a good prognosis for many months, low risk of bleeding, no thrombocytopenia and coagulation disorders, prolongation of DAPT can be considered using the general population formula, in justified cases over a year. This proposal seems particularly reasonable in the context of increased thrombogenicity and a greater risk of vascular events in cancer patients [13]. In all cases, the timing of DAPT should be decided carefully, with common sense and individual approach. After the possible implementation of such treatment, more intensive supervision and more frequent checks are recommended to assess the level of bleeding risk, which may change in a patient with cancer at any time.

Optimal pharmacotherapy of ACS in oncological patients should not differ from the routinely used and should include: DAPT, statin, $\beta$-blocker, angiotensin converting enzyme inhibitor (ACE-I) and AT-1 receptors antagonists (ARA). Such treatment has been shown to reduce cardiovascular events by $32 \%$ over 12 months of follow-up after ACS treated invasively in cancer patients [31, 32]. However, in most registries and retrospective observations, antiplatelet drugs, especially $\mathrm{P} 2 \mathrm{Y} 12$ inhibitors, statins, $\beta$-blockers, ACE-I/ARA, were used less frequently than in the general population $[6,31,33]$. This is due to the fact that cancer patients have many disorders considered probably too often to be absolute contraindications to the use of drugs, fear of side effects and often an urgent need to implement diagnostics and oncological treatment.

In terms of long-term therapy as part of secondary prevention after myocardial infarction, drug interactions are of great importance [14]. For example, paclitaxel, through a common metabolic pathway through the CPY2C8 isoenzyme, reduces the availability of simvastatin, atorvastatin and fluvastatin [34]. For the same reason, clopidogrel, as a prodrug, must be converted into the active form of the drug in the liver by enzymatic systems in which many oncological drugs are metabolized. Therefore, its availability, antiplatelet effect and clinical effect may be incomplete. Spironolactone has an affinity for androgen receptors, so it may interfere with hormonal re-tuning in some forms of breast cancer or even weaken hormone therapy.

Therefore, if it is necessary to use mineralocorticoid receptor antagonists, it seems rational to choose selective preparation eplerenone [35].

\section{TAKOTSUBO SYNDROME}

Takotsubo syndrome is defined as a typical picture of myocardial infarction with symptoms such as typical or atypical coronary pain, ischemic ECG changes, typical increase and/or decrease in cardiac troponins and the presence of transient segmental disorders of left ventricular contraction that do not correspond to their location changes in coronary arteries [36-38]. As a rule, about $90 \%$ of incidents occur in older women and are associated with a stress situation. This syndrome was first described in 1990. It is diagnosed relatively rarely, in $1-3 \%$ of STEMI cases in the general population $[39,40]$. Among women it accounts for 5-6\% of STEMI cases [41]. Takotsubo syndrome is much more common in cancer patients. It is found in $4 \%$ to $39 \%$ of patients in this group [42-46]. Takotsubo syndrome may occur in patients cured of cancer and patients with active cancer, especially during treatment. There is a very strong association of the takotsubo syndrome and cancer described in the literature. Observations presented in the literature suggest that this syndrome may precede cancer. Therefore the takotsubo syndrome could be treated such the idiopathic thromboembolic incidence as a'marker of the cancer disease' [47-49].

In cancer patients, this syndrome develops primarily in older women $(76 \%)$ in older age, especially with advanced disease. Usually the onset of symptoms appears soon after surgery, bone marrow transplantation, during chemotherapy [50].

Takotsubo syndrome has been reported during treatment with 5-fluorouracil (5-FU), capecitabine, cytarabine, axitinib, trastuzumab, combretastatin, suggesting a cause-effect relationship of these drugs [51-54]. The mechanism of the disease is unknown. 
There are indications that symptoms are caused by the coronary microcirculation disorders resulting from the endothelial dysfunction that is damaged by oncological drugs and excessive levels of catecholamines released during stress [55-59]. Dysfunction of the pericytes may also play an important role. Disturbed communication on the pericyte-endothelocytes-cardiomyocytes axis could also lead to coronary microcirculation failure, ischemia and myocardial necrosis [60].

Until recently, the course of this disease was thought to be mild and with good prognosis. However, recent observations indicate an unfavorable long-term prognosis. Long-term mortality is comparable to mortality in NSTEMI [61]. In patients with cancer and takotsubo syndrome more severe course is observed: more acute ECG changes, increase of brain natriuretic peptide (BNP) concentration, higher leukocytosis, higher CRP levels and higher total and cardiac mortality [42].

In order to diagnose takotsubo syndrome, it is needed to demonstrate transient regional contraction abnormalities disorders revealed in imaging (usually echocardiography) and the absence of coronary artery disorders responsible for them seen in imaging such as coronary angiography or contrast coronary tomography $[36,37]$.

There is no specific data about treatment. The therapeutic doses of anticoagulant are not recommended, except for the situation with the thrombus located in the enlarged, akinetic apex. The use of antiplatelet drugs is discussable while it seems reasonable to use $\beta$-blockers (multifunctional and vasodilatation) and ACE-I and ARA. A reduction mortality in annual follow-up was shown for ACE-I/ARA, but not for $\beta$-blockers [62].

\section{SUMMARY}

Acute coronary syndrome is a condition often coexisting with cancer. Both cancer and its treatment may be responsible for the formation of unstable atherosclerotic plaques and thrombus in the coronary arteries. Often in cancer patients. Often in cancer patients non-ST segment elevation myocardial infarction and type 2 myocardial infarction occur. From a clinical point of view it is important to understand the mechanism of oncological drugs responsible for ACS. One can distinguish three types of vascular toxicity. In the first type, the growth prevails atherosclerotic plaques and their destabilization due to the action of drugs; in the second type the primary mechanism is thrombus formation in the vessel; in the third the major mechanism is reversible vasoconstriction. The diagnosis of ACS among cancer patients is difficult and symptoms are often ambiguous. The interview is of great importance in the diagnosis, especially the history of previous disorders, type of cancer, stage and oncological drugs used.

Treatment depends on many factors modifying the clinical picture of ACS. An invasive strategy should always be considered and, if conservative treatment is selected, the decision should be based on the coexisting facts. Even with optimally selected management, oncological patients have a worse ACS prognosis compared to patients without cancer. Both invasive and conservative treatment is hindered by such problems as: thrombocytopenia, bleeding tendency, thrombogenicity, kidney failure and liver dysfunction, coexistence of comorbidities, usually older age of patients, frailty syndrome, the use of many anti-cancer drugs and adjuvants, urgent need of invasive diagnostic and therapeutic procedures. Two common forms of ACS in the group of oncological patients deserve special attention: vasoconstriction after 5-FU and its derivatives and takotsubo syndrome, which develops much more often in this group of patients than in the general population.

Treating ACS in a patient with cancer is a real clinical challenge. Very often it is difficult to choose the optimal treatment strategy. In this group of patients, individual decisions should be made, but always through the prism of recommendations for the general population.

\section{References}

1. Ibanez B, James S, Agewall S et al. ESC Scientific Document Group. 2017 ESC Guidelines for the management of acute myocardial infarction in patients presenting with ST-segment elevation: The Task Force for the management of acute myocardial infarction in patients presenting with ST-segment elevation of the European Society of Cardiology (ESC). Eur Heart J. 2018; 39(2): 119-77.

2. Roffi M, Patrono C, Collet JP et al. ESC Scientific Document Group. 2015 ESC Guidelines for the management of acute coronary syndromes in patients presenting without persistent ST-segment elevation: Task Force for the Management of Acute Coronary Syndromes in Patients Presenting without Persistent ST-Segment Elevation of the European Society of Cardiology (ESC). Eur Heart J. 2016; 37(3): $267-315$.

3. Andersen HR, Nielsen TT, Rasmussen K et al. DANAMI-2 Investigators. A comparison of coronary angioplasty with fibrinolytic therapy in acute myocardial infarction. N Engl J Med. 2003; 349(8): 733-42.

4. Potts JE, lliescu CA, Lopez Mattei JC et al. Percutaneous coronary intervention in cancer patients: a report on the prevalence and outcomes in the United States. Eur Heart J. 2019; 40: 1790-800. 
5. Guddati AK, Joy PS, Kumar G. Analysis of outcomes of percutaneous coronary intervention in metastatic cancer patients with acute coronary syndrome over a 10-year period. J Cancer Res Clin Oncol. 2016; 142: 471-9.

6. Yusuf SW, Daraban N, Abbasi N et al. Treatment and outcomes of acute coronary syndrome in the cancer population. Clin Cardiol. 2012; 35(7): 443-50.

7. Neumann FJ, Sousa-Uva M, Ahlsson A et al. [2018 ESC/EACTS Guidelines on myocardial revascularization]. Kardiol Pol. 2018; 76(12): 1585-1664

8. Giza DE, Boccalandro F, Lopez-Mattei J et al. Ischemic Heart Disease: Special Considerationsn Cardio-Oncology. Curr Treat Options Cardiovasc Med. 2017(5): 3-13.

9. Yadav PK, Bagur R, Baquero GA et al. Safety and Feasibility of Transradial Catheterization in Breast Cancer Survivors: A 2-Center International Experience. JACC Cardiovasc Interv. 2015; 8(4): 639-41.

10. Rao SV, Kedev S. Approaching the post-femoral era for coronary angiography and intervention. J Am Coll Cardiol Intv. 2015; 8: 524-6.

11. Lozano I, Batalla A, Rondan J et al. Radial Artery in Breast Cancer Survivors: A Possible Choice but Always With Caution. JACC Cardiovasc Interv. 2015; 8(9): 1275-6.

12. Iliescu CA, Grines CL, Herrmann J et al. SCAI Expert consensus statement: Evaluation, management, and special considerations of cardio-oncology patients in the cardiac catheterization laboratory (endorsed by the cardiological society of India, and sociedad Latino Americana de Cardiologia intervencionista). Catheter Cardiovasc Interv. 2016; 87(5): E202-23.

13. Giza DE, Marmagkiolis K, Mouhayar E. et al. Management of CAD in Patients with Active Cancer: the Interventional Cardiologists' Perspective. Curr Cardiol Rep. 2017; 19(6): 56-66.

14. Banasiak W, Zymliński R, Undas A. Optimal management of cancer patients with acute coronary syndrome. Pol Arch Intern Med. 2018; 128(4): 244-53.

15. Mohanty BD, Mohanty S, Hussain $Y$ et al. Management of ischemic coronary disease in patients receiving chemotherapy: anuncharted clinical challenge. Future Cardiol. 2017; 13(3): 247-57.

16. Krone RJ. Managing coronary artery disease in the cancer patient. Prog Cardiovasc Dis. 2010; 53(2): 149-56.

17. Elting LS, Rubenstein EB, Martin CG et al. Incidence, cost, and outcomes of bleeding and chemotherapy dose modification among solid tumor patients with chemotherapy-induced thrombocytopenia. J Clin Oncol. 2001; 19(4): 1137-46.

18. Iliescu C, Durand JB, Kroll M. Cardiovascular interventions in thrombocytopenic cancer patients. Tex Heart Inst J. 2011; 38(3): 259-60.

19. Gross CM, Posch MG, Geier C et al. Subacute coronary stent thrombosis in cancer patients. J Am Coll Cardiol. 2008; 51(12): 1232-3.

20. Mauri L, Yeh RW, Kereiakes DJ. Duration of dual antiplatelet therapy after drug-eluting stents. N Engl J Med. 2015; 372(14): 1373-4

21. Iliescu CA, Cilingiroglu M, Giza DE et al. "Bringing on the light" in a complex clinical scenario: Optical coherence tomography-guided discontinuation of antiplatelet therapy in cancer patients with coronary artery disease (PROTECT-OCT registry). Am Heart J. 2017; 194: 83-91.

22. Khakoo AY, Yeh ET. Therapy insight: management of cardiovascular disease in patients with cancer and cardiac complications of cancer therapy. Nat Clin Pract Oncol. 2008; 5: 655-67.

23. Hong RA, Limura T, Sumida KN et al. Cardio-oncology/Onco-kardiology. Clin Cardiol. 2010; 33: 733-7.

24. Feher A, Kampaktsis PN, Parameswaran R et al. Aspirin Is Associated with Improved Survival in Severely Thrombocytopenic Cancer Patients with Acute Myocardial Infarction. Oncologist. 2017; 22(2): 213-21.

25. Wallentin L, Becker RC, Budaj A et al. PLATO Investigators. Ticagrelor versus clopidogrel in patients with acute coronary syndromes. N Engl J Med. 2009; 361(11): 1045-57.

26. Wiviott SD, Braunwald E, McCabe CH et al.; TRITON-TIMI 38 Investigators. Prasugrel versus clopidogrel in patients with acute coronary syndromes. N Engl J Med. 2007; 357(20): 2001-15

27. Serebruany VL. Aggressive chronic platelet inhibition with prasugrel and increased cancer risks: revising oral antiplatelet regimens? Fundam Clin Pharmacol. 2009; 23: 411-7.

28. Mega JL, Braunwald E, Wiviott SD et al.; ATLAS ACS 2-TIMI 51 Investigators. Rivaroxaban in patients with a recent acute coronary syndrome. N Engl J Med. 2012; 366(1): 9-19.

29. Key NS, Khorana AA, Kuderer NM et al. Venous Thromboembolism Prophylaxis and Treatment in Patients With Cancer: ASCO Clinical Practice Guideline Update. J Clin Oncol. 2020; 38(5): 496-520.

30. Yusuf S, Zhao F, Mehta SR et al. Effects of Clopidogrel in Unstable Angina to Prevent Recurrent Events Trial Investigators. Effects of clopidogrel in addition to aspirin in patients with acute coronary syndromes without ST-segment elevation. N Engl J Med. 2001; 345(7): 494-502.

31. lannaccone M, D'Ascenzo F, Vadalà P et al. Prevalence and outcome of patients with cancer and acute coronary syndrome undergoing percutaneous coronary intervention: a BleeMACS substudy. Eur Heart J Acute Cardiovasc Care. 2017; 7: 631-8.

32. Iannaccone M, D'Ascenzo F, De Filippo $\mathrm{O}$ et al. Optimal medical therapy in patients with malignancy undergoing percutaneous coronary intervention for acute coronary syndrome: a BleeMACS SubStudy. Am J Cardiovasc Drugs. 2017; 17: 61-71.

33. Gong IY, Yan AT, Ko DT et al. Temporal changes in treatments and outcomes after acute myocardial infarction among cancer survivors and patients without cancer, 1995 to 2013. Cancer. 2018; 124: 1269-78.

34. Tornio A, Pasanen MK, Laitila J et al. Comparison of 3-hydroxy-3-methylglutaryl coenzyme a (HMG-CoA) reductase inhibitors (statins) as inhibitors of cytochrome P450 2C8. Basic Clin Pharmacol Toxicol. 2005; 97: 104-8.

35. Pitt B. Effect of aldosterone blockade in patients with systolic left ventricular dysfunction: implications of the RALES and EPHESUS studies. Mol Cell Biol. 2004; 217: 53-8.

36. Ono R, Falcão LM. Takotsubo cardiomyopathy systematic review: Pathophysiologic process, clinical presentation and diagnostic approach to Takotsubo cardiomyopathy. Int J Cardiol. 2016; 209: 196-205.

37. Ghadri JR, Wittstein IS, Prasad A et al. International Expert Consensus Document on Takotsubo Syndrome (Part I): Clinical Characteristics, Diagnostic Criteria, and Pathophysiology. Eur Heart J. 2018; 39(22): 2032-46.

38. Ghadri JR, Wittstein IS, Prasad A et al. International Expert Consensus Document on Takotsubo Syndrome (Part II): Diagnostic Workup, Outcome, and Management. Eur Heart J. 2018; 39(22): 2047-62.

39. Prasad A, Dangas G, Srinivasan M et al. Incidence and angiographic characteristics of patients with apical ballooning syndrome (takotsubo/stress cardiomyopathy) in the HORIZONS-AMI trial: an analysis from a multicenter, international study of ST-elevation myocardial infarction. Catheter Cardiovasc Interv. 2014; 83: 343-8.

40. Bybee KA, Prasad A, Barsness GW et al. Clinical characteristics and thrombolysis in myocardial infarction frame counts in women with transient left ventricular apical ballooning syndrome. Am J Cardiol. 2004; 94: 343-6.

41. Redfors $B$, Vedad $R$, Angeras $O$ et al. Mortality in takotsubo syndrome is similar to mortality in myocardial infarction - a report from the SWEDEHEART registry. Int J Cardiol. 2015; 185: 282-9.

42. Girardey M, Jesel L, Campia U et al. Impact of Malignancies in the Early and Late Time Course of Takotsubo Cardiomyopathy. Circ J. 2016; 80(10): 2192-8.

43. Burgdorf C, Kurowski V, Radke PW. Long-term prognosis of transient left ventricular ballooning syndrome and cancer. Heart Lung. 2011 ; $40: 472$.

44. Burgdorf $\mathrm{C}$, Kurowski V, Bonnemeier $\mathrm{H}$ et al. Long-term prognosis of the transient left ventricular dysfunction syndrome (Tako-Tsubo cardiomyopathy): Focus on malignancies. Eur J Heart Fail. 2008; 10: 1015-9.

45. Behr ER, Mahida S. Takotsubo cardiomyopathy and the long-QT syndrome: an insult to repolarization reserve. Europace. $2009 ; 11: 697-700$.

46. Madias JE. Transient attenuation of the amplitude of the QRS complexes in the diagnosis of Takotsubo syndrome. Eur Heart J Acute Cardiovasc Care. 2014; 3: 28-36. 
47. El-Sayed AM, Brinjikji W, Salka S. Demographic and comorbid predictors of stress (takotsubo) cardiomyopathy. Am J Cardiol. 2012; 110(9): 1368-72.

48. Sattler K, El-Battrawy I, Lang S et al. Prevalence of cancer in Takotsubo cardiomyopathy: short and long-term outcome. Int J Cardiol. $2017 ; 238$ : $159-65$.

49. Giza DE, Lopez-Mattei J, Vejpongsa P et al. Stress-induced cardiomyopathy in cancer patients. Am J Cardiol. 2017; 120(12): 2284-8.

50. Vejpongsa P, Banchs J, Reyes M et al. Takotsubo cardiomyopathy in cancer patients: triggers, recovery, and resumption of therapy. J Am Coll Cardiol. 2015; 65(10S): A927.

51. Franco TH, Khan A, Joshi V et al. Takotsubo cardiomyopathy in two men receiving bevacizumab for metastatic cancer. Ther Clin Risk Manag. 2008; 4(6): 1367-70.

52. Numico G, Sicuro M, Silvestris N et al. Takotsubo syndrome in a patient treated with sunitinib for renal cancer. J Clin Oncol. 2012; 30(24): e218-e20.

53. Baumann S, Huseynov A, Goranova D et al. Takotsubo cardiomyopathy after systemic consolidation therapy with high-dose intravenous cytarabine in a patient with acute myeloid leukemia. Oncol Res Treat. 2014; 37(9): 487-90.

54. Ovadia D, Esquenazi Y, Bucay M et al. Association between takotsubo cardiomyopathy and axitinib: case report and review of the literature. J Clin Oncol. 2015; 33(1): e1-e3.

55. Polk A, Vistisen K, Vaage-Nilsen M et al. A systematic review of the pathophysiology of 5-fluorouracil-induced cardiotoxicity. BMC Pharmacol Toxicol. 2014; 15: 47.

56. Stewart T, Pavlakis N, Ward M. Cardiotoxicity with 5-fluorouracil and capecitabine: more than just vasospastic angina. Intern Med J. 2010; 40(4): 303-7.

57. Grunwald MR, Howie L, Diaz LA et al. Takotsubo cardiomyopathy and Fluorouracil: case report and review of the literature. J Clin Oncol. 2012; 30(2): e11-e4.

58. Smith SA, Auseon AJ. Chemotherapy-induced takotsubo cardiomyopathy. Heart Fail Clin. 2013; 9(2): $233-42$.

59. Kobayashi N, Hata N, Yokoyama S et al. A case of Takotsubo cardiomyopathy during 5-fluorouracil treatment for rectal adenocarcinoma. J Nippon Med Sch. 2009; 76(1): 27-33.

60. Sen F, Yildiz I, Basaran M et al. Impaired coronary flow reserve in metastatic cancer patients treated with sunitinib. J BUON. 2013; 18(3): 775-81.

61. Redfors B, Vedad R, Angerås $\mathrm{O}$ et al. Mortality in Takotsubo syndrome is similar to mortality in myocardial infarction - A report from the SWEDEHEART registry. Int J Cardiol. 2015; 185: 282-9.

62. Templin C, Ghadri JR, Diekmann J et al. Clinical Features and Outcomes of Takotsubo (Stress) Cardiomyopathy. N Engl J Med. $2015 ; 373: 929-38$.

Conflict of interests: Author declare no conflict of interest.

Financial support: There was no financial support.

Ethics:

The authors had full access to the data and take full responsibility for its integrity. All authors have read and agreed with the content of the manuscript as written. The paper complies with the Helsinki Declaration, EU Directives and harmonized requirements for biomedical journals. 